\title{
Handling Equipment Allocation Optimization of Railroad-highway Combined Transportation: Bi-Objective Model and MEACO Algorithm
}

\author{
Qi Zhang, Yuan Ye, Li Zhang, and Hao Yang
}

\begin{abstract}
Coordinating connection between railroad and highway in multimodal transportation is very complex and important. It is concerned with the time and cost consuming of containers, the operation efficiency, and resources allocation of railroad and highway. This paper studies the equipment allocation optimization in railway container center station. It establishes a bi-objective optimization model to solve the problem. The two objectives of the model are getting the minimum daily comprehensive costs of equipments, and getting the minimum dwelling time of railway container flatcars on loading and unloading line. Allocating more equipment will reduce the dwelling time obviously. But there are some risks of idle equipment and high costs, too. These are two irreconcilable objectives. The MEACO algorithm is used to solve this problem making Chongqing container center station as an example. The model is verified by actual data. The results suggest the optimal allocation of the handling equipments and are in accordance with the fact of the station.
\end{abstract}

Index Terms-Bi-objective optimization model, handling equipment allocation, MEACO algorithm, railroad-highway combined transportation

\section{INTRODUCTION}

$\mathrm{S}$ MOOTH connection could reduce the time and cost consuming in the transportation process. The advantage and efficiency of railroad-highway combined transportation require much for connecting effectively of these two different transportation modes. Reducing the loading and unloading business work and dwelling time seems to be a good measure.

Railway container center station is the joint hub in the railroad-highway combined transportation. There are a lot of loading and unloading businesses in the station. Handling

Manuscript submitted on October 31, 2013. This work was supported in part by China Railway Corporation (2012X012-H), and Fundamental Research Funds for the Central Universities (2011JBM061).

Qi Zhang. Associate professor, School of Traffic \& Transportation, Beijing Jiaotong University, Beijing, 100044, China (1-662-518-1762 or +86-18600231123; e-mail: qizhang@bjtu.edu.cn).

Yuan Ye. Xiamen airport logistics management Co. Ltd, No.95 Xiangyun Rd, Huli District, Xiamen, 361000, China (email: yeyuan.lala@163.com).

Li Zhang. Associate professor, Department of Civil and Environmental Engineering, Mississippi State University, Starkville, MS39762, USA (e-mail: lzhang@cee.msstate.edu).

Hao Yang. Professor, School of Traffic \& Transportation, Beijing Jiaotong University, Beijing, 100044, China (e-mail: hyang@bjtu.edu.cn). equipments are the machine both for such work and the crucial conjunction to connect the railroad and highway. Thus, one important problem needs to be settled is to select properly and allocate reasonably the equipments. There are at least two major aspects need to be considered in the equipment selection and allocation problem, ESAP. One is the machine type. It must be high efficient and be suitable for what to do. Another is the machine amount. It must be enough and reasonable, either meeting the business demand or without much idleness.

\section{LITERATURE REVIEW}

In recent years, many researchers are focusing on the equipment selection and allocation problem. Some researchers are concerned with the most suitable equipment selection in railway stations, such as Song et al. (2005), Bai et al. (2002), Ma (2005), Li et al. (2005). Some researchers are concerned with the tusk allocation basing on the equipment selection, such as Murty K G (2000), Linn R (2003). Some researchers are concerned with the amount decision problem of railway container station, for example Liang et al. (2009), Huo et al. (2006), Wong (2008), Li et al. (2009). Comparatively, most advanced researches are concerned with the equipments allocation problem of harbor and dock, such as Yang (1995), CF Daganzo (1989), R. I. Peterkofsky \& CF Daganzo (1990), J. B. Tabernacle (1995), J. Bose et al. (2000), Lai K.K \& Lam K (1994), Kozan E \& Preston P (1999), CF Daganzo (1989), Pyung Hoi Koo (2004), Hao (2003), Ji et al. (2010), Yong et al. (2008).

Researchers applied different methods and developed different models, from various research points, to solve the equipment selection and allocation problem. The research results are abundant, especially in the aspects of harbor and dock.

Bai et al. (2002) studied the alternative types of handling equipments which were likely to be suitable for the railway container station. The authors studied the yard utilization rate of each kind of equipments adopting the unit-acreage. Basing on the operation costs by the whole life cycle input-output method (WLCIO), the authors gave the optimal selection of equipment type as Rail Mounted Gantry Crane (RMGC).

Liang et al. (2009) combined the discrete event simulation principle (DESP) with event graph method (EG/ED) to develop the simulation model. The authors divided the trailers into two 
types, the inner trailer operated by railroad yard and the outer trailer operated by the highway transportation corporations. With the simulation of outer trailer randomly arrival, the authors obtained the optimal allocation of transferring resources, including the inner trailers and the loading and unloading equipments.

Basing on the research results of equipment type selection, Linn R (2003) solved the tusk allocation problem by proposing mixed integer programming (MIP). The authors got the working sequence and time scheduling of RMGC between different container yards.

R. I. Peterkofsky and CF Daganzo (1990) combined a mathematical programming model with an allocation strategy to solve the static Quayside Container Crane Allocation Problem (QCCAP). The authors calculated the maximum throughput of one berth in rush hour aiming to reduce the waiting fee and dwelling time of container ship. While J. B. Tabernacle (1995) considered the Quayside Container Crane (QCC) as parallel business and transferred the QCCAP into Open Shop Scheduling Problem (OSSP). The authors developed an integer programming model and applied the branch and bound method to solve the problem.

CF Daganzo (1989) formed an integer programming model and designed an optimal inventory strategy to obtain an optimal RMGC business plan. The programming model aimed for the highest efficiency of loading and unloading business of all RMGCs allocated to work for the specific container ship.

Ji et al. (2010) developed a Shortest Path Problem (SPP) optimal model from the point of view of simultaneous business between in-port and ex-port container ships. The authors applied the POEM optimal platform and used the simulative data to get results. According to the results, with the constraints of minimum waiting time of Quayside Container Crane, the authors estimated the optimal amount of trailers matching the working plan of QCC.

This paper focused its studies on the loading and unloading equipment amount decision in railway container center station. Based on the problem description and literature review above, the main contributions of this paper are described as follows.

- The paper considers the core of the seamless connection in railroad-highway combined transportation is the coordination between different businesses, including railway container flatcars, handling equipments and highway trailers or chassis. The paper makes the coordinative connection between those businesses as objective to study the Handling Equipment Allocation Problem (HEAP).

- A bi-objective model stresses the coordination between different businesses by daily comprehensive costs of equipments and dwelling time of rail flatcars. Business efficiency and operation costs are the constraints of the model. MEACO algorithm is represented to search for the optimal results.

Basing on the factual of Chongqing railway container center station, the model is verified to be useful. And the optimal equipment allocation is given by the results of the model.

\section{Bi-objective Optimal Model of Handling Equipment ALLOCATION}

The handling equipment allocation in railway container center stations should meet the demand of timeliness of container transportation and the requirement of operation benefits of station at the same time. This paper developed a bi-objective optimal model with the bounds from both daily comprehensive costs and dwelling time, making the equipment amount as its decision variable.

\section{A. Daily comprehensive costs of handling equipments $\mathrm{Cg}$}

Basing on the relevant research results, this paper considered the main equipment of loading and unloading in railway container center station is Rail Mounted Gantry Crane (RMGC). Daily comprehensive costs of this machine should include some kinds of costs, shown as table I.

TABLE I

COST CATEGORIES OF RMGC

\begin{tabular}{ccc}
\hline \hline & Fixed Costs & Variable Costs \\
\hline \multirow{3}{*}{ Cost categories } & $\begin{array}{c}\text { depreciation funds } \\
\text { major repair funds } \\
\text { acquisition cost }\end{array}$ & $\begin{array}{c}\text { daily maintenance cost } \\
\text { fuel and electric power cost } \\
\text { cost of labor }\end{array}$ \\
\hline \hline
\end{tabular}

There are some matching facilities for the operation of RMGC, such as the loading and unloading lines, hard-surface pavement of yards, and the running lines of RMGC. Then, there are the corresponding costs arising.

Daily fixed costs $f_{j}$ of the $j^{\text {th }}$ RMGC is shown as (1).

$$
f_{j}=c_{f} \cdot \frac{(1-i)}{N \times d}+\frac{c_{M}}{d}
$$

In equation (1), $c_{f}$ is the acquisition cost of each RMGC. $c_{M}$ is the average annual maintenance cost of each RMGC. $N$ is the depreciation period and $i$ is the residual value of the machine. The last $d$ is the working day of the railway container center stations.

Daily operation costs $c_{v j}$ of the $j^{\text {th }}$ RMGC is defined in (2).

$$
c_{v j}=c_{l}+Q_{j} \times c_{m}+c_{p} \times T_{j}
$$

In equation (2), $c_{l}$ is the daily cost of labor of each RMGC. $Q_{j}$ is the total number of containers loaded or unloaded by the $j^{\text {th }}$ machine in one day. The average maintenance cost of each TEU is $c_{m}$. The electric power cost of RMGC per hour is $c_{p}$. $T_{j}$ is the average daily working time of the $j^{\text {th }}$ RMGC.

Construction costs of matching facilities $C_{b}$ is shown as (3).

$$
C_{b}=\left(c_{r}+c_{c r}+c_{s}\right) \times \frac{(1-i)}{N \times d} \times r
$$

There are three kinds of costs in (3). Infrastructural construction costs of each railway loading and unloading line is $c_{r}$. Construction cost of running line is $c_{r s}$. Hard-pavement cost 
of working areas and yards is $c_{s}$. $N$ is the depreciation periods of these infrastructures and $i$ is the residual value. The number of loading and unloading lines matching with the amount allocation of RMGC is defined as $r$.

Then, the average daily costs of handling equipment $C_{g}$ can be defined as (4).

$$
C_{g}=\xi \cdot\left[\sum_{j=l}^{m_{1}}\left(f_{j}+c_{v j}\right)+C_{b}\right]+(1-\xi) \cdot\left[\sum_{j=l}^{m_{2}}\left(f_{j}^{\prime}+c_{v j}^{\prime}\right)+C_{b}^{\prime}\right]
$$

As illustrated in equation (4), $m_{1}$ and $m_{2}$ are the numbers of RMGC and Front Crane respectively. The $\xi$ is a coefficient. If $\xi=1$, the equipment selection decision will be the RMGC. Then, it will be the Front Crane. If the equipment selection decision is the Front Crane in railway container center station, the $f_{j}^{\prime}, c_{v j}$ ' and $C_{b}$ 'will be the daily fixed costs, daily operation costs and construction costs of matching facilities of the Front Crane respectively. And $f_{j}^{\prime}$ and $c_{v j}$ ' have the same expressions as (1) and (2), but $C_{b}$ is shorten because of without the infrastructural construction costs of running line, $c_{r s}$.

\section{B. Average dwelling time on loading and unloading lines of} railroad flatcars $\bar{T}$

Dwelling time of railroad flatcars on loading and unloading lines is consisted of two parts, the loading and unloading time $T$ and the waiting time $W$. The average dwelling time on loading and unloading lines of railroad flatcars $\bar{T}$ is expressed as (5).

$$
\bar{T}=\left[\sum_{j=1}^{m_{3}}\left(T_{j}+W_{j}\right)\right] / m_{3}
$$

In the equation (5), loading and unloading time of the $j^{\text {th }}$ railroad flatcars is $T_{j}$, and their waiting time is $W_{j}$. And $m_{3}$ is the number of railroad flatcars entering the lines during the operation period of the railway container center station.

Getting the dwelling time of railroad flatcars under different equipment selection decision will be helpful for analyzing the efficiency change caused by the technology characteristics of loading and unloading equipments. Thus, the paper makes the dwelling time as one of its objective function.

\section{Bi-objective optimal model}

(1) Definitions of the bi-objective optimal problem

When the two objectives in the optimal model are all the minimum objectives, the problem could be expressed as (6).

$$
\min F(x)=\min \left(f_{1}(x), f_{2}(x)\right)
$$

According to the multi-objective theory, the paper proposed some definitions about the bi-objectives.

Definition1. It is a kind of Pareto dominance relationship. For the solution $x_{1}, x_{2} \in \Omega, x_{1}$ dominates $x_{2}$. When and only when exists $\forall i \in\{1,2\}$, it gets $f_{i}\left(x_{1}\right) \leq f_{i}\left(x_{2}\right)$. And it exists $\exists i \in\{1,2\}$, it gets $f_{i}\left(x_{1}\right)<f_{i}\left(x_{2}\right)$. Then, the equation $x_{1} \succ x_{2}$ is defined as $x_{1}$ dominates $x_{2}$.
Definition2. It is a Pareto optimal solution or a non-dominance solution. When and only when the solution $x \in \Omega$ does not exist and there is $x \succ x^{*}$, the solution $x^{*} \in \Omega$ is called as the Pareto optimal solution.

Definition3. It is a Pareto optimal set. The set of the Pareto optimal solutions for the bi-objective optimal problem is called as the Pareto optimal set. It is defined as $P^{*}=\left\{x^{*} \in \Omega \mid \neg \exists x \in \Omega: x \succ x^{*}\right\}$.

Definition4. It is the real Pareto front surface. Given an optimal solution set of a bi-objective optimal problem $P^{*}$. The curved surface reflected by all the Pareto optimal solutions in the objective space is called as the real Pareto front surface. It is defined as $P F^{*}=\left\{F\left(x^{*}\right)=\left(f_{1}\left(x^{*}\right), f_{2}\left(x^{*}\right) \mid x^{*} \in P^{*}\right)\right\}$.

(2) Bi-objective optimal model of handling equipment allocation

According to the problem description above, the two minimum objectives of the bi-objective optimal model are the daily comprehensive costs of handling equipments $C_{g}$ and the dwelling time of railway container flatcars on loading and unloading lines $\bar{T}$. The bi-objective optimal modal of handling equipment allocation is expressed as (7).

$$
\min F(x)=\min \left(C_{g}, \bar{T}\right)
$$

\section{ALGORITHM FOR THE BI-OBJECTIVE OPTIMAL MODEL OF HANDLING EQUIPMENT ALLOCATION}

\section{A. MEACO algorithm for bi-objective optimal model}

This paper studied the container handling equipment allocation problem based on the railroad-highway seamless conjunction. The timeliness is the key for this problem. Then, in the objective function, $\bar{T}$ is more important than $C_{g}$. In applying the MEACO to solve the problem, the paper transferred the $C_{g}$ into the constraint of $\min \bar{T}$, the objective function. Then the bi-objective optimal problem, as (7), is transferred into a single objective problem with optimal constraints, shown as (8).

$$
\begin{gathered}
\min \bar{T} \\
\text { s.t. } C_{g}\left(m_{i}\right)_{\min } \leq C_{g}\left(m_{i}\right) \leq C_{g}\left(m_{i}\right)_{\text {max }} \\
m_{i} \in D
\end{gathered}
$$

As in equation (8), $D$ is the domain of decision variables. It is defined as $m_{i} \geq 0, m_{i} \in Z^{n}, Z^{n}$ means $n$-dimensional integer set.

\section{B. Decision variables notations and values range}

Daily comprehensive costs are determined by the acquisition costs of the equipments, the infrastructure construction costs of the matching facilities and the daily operation costs. Then, one important variable is the amount of RMGC, $m_{l}$.

The value range of $m_{l}$ is concerned with the container throughput of the railway container center station, the number and the valid length of loading and unloading lines of the station. In general speaking, $m_{l}$ is concerned with the daily total volume of loading and unloading business $Q$ from the point of view of 
throughput. The value of $m_{l}$ is no less than the demand of 24-hour continuously business. In China, the standard valid lengths of loading and unloading lines are $850 \mathrm{~m}$ and $1050 \mathrm{~m}$ in railway container center station. The running distance of RMGC is about $250 \mathrm{~m}$ to $350 \mathrm{~m}$ ( $\mathrm{Yu}$, et al., 2005). Then the amount of RMGC allocated will be up to 4 in 850-meter line and up to 5 in 1050-meter line. It is supposed that the number of loading and unloading lines in the railway container center station is $r$. The amount range of RMGC allocated could be expressed as (9).

$$
\begin{aligned}
& Q /(24 \cdot v) \leq m \leq 4 r \ldots L=850 m \\
& Q /(24 \cdot v) \leq m \leq 5 r \ldots L=1050 m
\end{aligned}
$$

In equation (9), $v$ is the average rate of loading and unloading of RMGC. The value of $v$ is $30 T E U / h$, and $m \in Z$.

It is necessary to say that under some conditions, $m$ is not determined by $r$ thoroughly. The amount of RMGC allocated is $m$. This allocation plan could meet the demand of loading and unloading business. While the number of loading and unloading lines is added up to $r^{\prime}, r^{\prime}>r$. According to (9), $m$ needs to be added up to $m^{\prime}, m^{\prime}>m$. Then the number of $\left(m^{\prime}-m\right)$ RMGC will be in idle state. In order to get the minimum operation costs of equipments, the amount of RMGC allocated will be kept at the level of $m$.

\section{Notations and values of time parameters in objective function}

The loading and unloading time $T_{j}$ and the waiting time on line $W_{j}$ are concerned with some factors, including the interval time of railway flatcars arrival, the number of loading and unloading lines of the station, as well as the amount of RMGC allocated for each line. Those RMGCs on the same loading and unloading line are working together or cooperatively. They are interdependent. At the same time, different RMGCs on different lines work respectively. They are mutual independent. Thus we considered all RMGCs allocated to a single line as one service desk while applying the Queuing Theory to simulate the loading and unloading business process in railway container center station.

But the amount of RMGC allocated for different lines maybe different. It causes the different business time in different line. It means that the service time servicing one customer of each service desk maybe different. This is not accordance with the Queuing Theory. Then we developed the theory to calculate the $T_{j}$ and $W_{j}$ by using the ratio between the number of loading and unloading lines $r$ and the amount of the RMGCs $m$.

(1) Judgment of $r$ and $m$.

In order to coordinate the connection between railroad and highway in container transportation, the waiting time $W_{j}$ should be cut down to zero as far as possible. When $W_{j}>0$, there will be a queue. The length of the loading and unloading line is limited. And the length of the waiting queue is limited, as (10).

$$
N \leq r \cdot L_{r} / L_{t s}
$$

In equation (10), $N$ is the maximum number of railway flatcars waiting on the loading and unloading lines, $r$ is the number of loading and unloading lines in the station, $L_{r}$ is the valid length of loading and unloading line, and $L_{t s}$ is the average length of each flatcars.

While the number of railway flatcars waiting on the lines is up to $N$, the next group will be rejected into the lines. While the groups entering on the lines need to wait, and this waiting makes the $P_{N}>0, P_{N}$ is the possibility of the number of waiting flatcars up to N. It means that the amount of RMGC allocated cannot meet the demand of loading and unloading business work. $P_{N}$ could be obtained by applying the Queuing Theory based on the following assumptions.

- Assumption1. Considering each loading and unloading line as a service desk, then the number of service desks $c$ is the same as the number of the lines $r$.

- Assumption2. The interval time of each flatcar's arrival yields to the Poisson distribution.

- Assumption3. The loading and unloading businesses on different lines are mutual independent. Each line is allocated the same number of RMGCs.

Then, it can be considered as a service system with limited

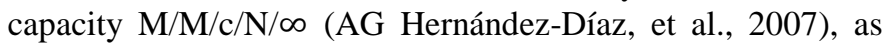
(11).

$$
\begin{aligned}
& p_{0}=\left[\sum_{k=0}^{c} \frac{(c \rho)^{k}}{k !}+\frac{c^{c}}{c !} \cdot \frac{\rho\left(\rho^{c}-\rho^{N}\right)}{1-\rho}\right]^{-1}, \rho \neq 1 \\
& p_{n}=\left\{\begin{array}{l}
\frac{(c \rho)^{\rho}}{n !} p_{0}, 0 \leq n \leq c \\
\frac{c^{c}}{c !} \rho^{n} p_{0}, c \leq n \leq N
\end{array}\right. \\
& \rho=\lambda / c u
\end{aligned}
$$

(2) $T_{j}$ and $W_{j}$.

If the station has only one line, the loading and unloading business time $T_{j}$ and waiting time for loading and unloading on line $W_{j}$ are calculated by (12).

$$
\begin{aligned}
T_{j} & =\left\{\begin{array}{c}
\left(\left[\delta / m_{i}\right]+1\right) \cdot 60 / v_{i},\left[\delta / m_{i}\right] \notin Z \\
{\left[\delta / m_{i}\right] \cdot 60 / v_{i},\left[\delta / m_{i}\right] \in Z}
\end{array}\right. \\
W_{j} & =\max \left(T_{j}-\lambda, 0\right)
\end{aligned}
$$

As illustrated in equation (12), $\delta$ is the number of containers loaded by each railway container flatcars, $v_{i}$ is the average rate of loading and unloading business of each RMGC, $m_{i}$ is the amount of RMGCs allocated to a single loading and unloading line, and $\lambda$ is the interval time of railway container flatcars arrival.

\section{OPTIMAL MODEL VERIFICATION AND EXAMPLE}

This paper used the data from Chongqing railway container center station to testify the bi-objective model. 
Chongqing railway container center station was put into operation in December 2009. It took the container transportation around Chongqing hub, as well as relevant logistics businesses radiating all over China. The first term construction finished one loading and unloading line with valid length of $780 \mathrm{~m}$. The station was allocated 3 RMGCs in container yard. The running line of RMGC is $790 \mathrm{~m}$. In the long term, the station will have 8 loading and unloading lines. It can hold 4 trains to be loaded or unloaded simultaneously in its yard.

\section{A. The values of parameters in objective function}

There are many parameters involved in calculating the daily comprehensive costs $C_{g}$. This paper selected some important ones and listed them in table II.

TABLE II

THE VALUES OF PARAMETERS IN OBJECTIVE FUNCTION

\begin{tabular}{lll}
\hline \hline Parameters & Unit & RMGC \\
\hline Acquisition costs $c_{f i}$ & $\begin{array}{l}\text { ten-thousand } \\
\text { Yuan/each }\end{array}$ & 1000 \\
Annual major repair funds $c_{M i}$ & ten-thousand Yuan & 37.5 \\
Daily costs of labor $c_{l i}$ & Yuan/day & 450 \\
Infrastructural construction costs of & ten-thousand & 879.27 \\
running line $c_{c r}$ & Yuan/per line & \\
Hard-surface pavement costs of yard $c_{s i}$ & ten-thousand & 1185 \\
& Yuan/per line & \\
$\begin{array}{l}\text { Depreciation period of running line } N \\
\text { Depreciation period of hard-surface } \\
\text { yard } N\end{array}$ & year & year \\
Residual value $i$ & & 30 \\
$\begin{array}{l}\text { Depreciation period of handling } \\
\text { equipments } N_{i}\end{array}$ & year & 30 \\
$\begin{array}{l}\text { Construction costs of loading and } \\
\text { unloading line } c_{r}\end{array}$ & ten-thousand & 3120 \\
$\begin{array}{l}\text { Annual working day of equipments } d \\
\text { Power and electricity costs of }\end{array}$ & Yuan/per line & \\
equipments $c_{p i}$ & day & 360 \\
$\begin{array}{l}\text { Maintenance costs of equipments } c_{m i} \\
\text { Daily container volume of loading and } \\
\text { unloading } Q\end{array}$ & Yuan/hour & 73.36 \\
\hline \hline
\end{tabular}

According to a great deal of observation, the arrival process of container flatcars is a random dynamic process. It yields to Markov chain and follows some rules in arrival interval and amount. The amount of flatcars is about 6 TEU entering the loading and unloading lines every 5 minutes. To simplify the model, the paper assumed that the process yields to a normal distribution with $\delta=6 \mathrm{TEU}$. Then, the interval time of flatcars arrival is $\lambda_{l}=5 \mathrm{~min}$. Shown as in table3, the daily container volume of loading and unloading in Chongqing container center station is 1334TEU/day. Then, the average flatcars arriving at the loading and unloading lines per day are 223 groups according to the assumption above.

\section{B. The amount of RMGC allocated}

(1) Value range of objective function $m$.
The main handling equipment of Chongqing railway container center station is Railway Mounted Gantry Crane. The amount of the equipment allocated is not equal to zero. The valid length of loading and unloading line is $780 \mathrm{~m}$. According to (9), the value range of $m$ is $2<m<4 r$.

(2) Calculation of objective function $C_{g}$.

The constraints of single objective function are in (13).

$$
\begin{aligned}
& r \geq 1 \\
& 2 \leq m \leq 4 r \\
& r, m \in Z^{n}
\end{aligned}
$$

According to the (1), (2), (3) and (4), we can calculate the value of $C_{g}$. Because the handling equipment is RMGC, the $\xi=1$. The calculation results of $C_{g}$ are shown as table III.

TABLE III

TIME AND COSTS PARAMETERS OF DIFFERENT ALLOCATION PLANS OF RMGC

\begin{tabular}{lllccccccc}
\hline \hline 1 & 2 & 3 & 4 & 5 & 6 & 7 & 8 & 9 & 10 \\
line & RMGC & & TEU & hour & hour & Yuan & Yuan & Yuan & Yuan \\
\hline 1 & 3 & -- & 445 & 14.87 & 18.58 & 7083.3 & 4560.2 & 18255.3 & 29898.9 \\
& 4 & $3: 1$ & 445 & 14.87 & 18.58 & 9444.4 & 9120.5 & 19795.9 & 38360.9 \\
2 & 5 & $3: 2$ & 445 & 14.87 & 18.58 & 11805.6 & 9120.5 & 18705.3 & 39631.4 \\
& 6 & $3: 3$ & 445 & 14.87 & 18.58 & 14166.7 & 9120.5 & 19155.3 & 42442.5 \\
$\ldots$ & $\ldots$ & $\ldots$ & $\ldots$ & $\ldots$ & $\ldots$ & $\ldots$ & $\ldots$ & $\ldots$ & $\ldots$ \\
\hline \hline
\end{tabular}

As illustrated in table III, parameter1 is the number of loading and unloading lines in Chongqing railway container center station. Parameter2 is the total amount of RMGCs. Parameter 3 is the ratio between the number of the lines and the amount of the equipments allocated. Parameter4 is the daily volume of container to be loaded or unloaded by each RMGC in line1. Parameter5 is the average daily time of loading and unloading business of each RMGC in the line*. Parameter6 is the average daily operation time of power and electricity of each RMGC in line*. Parameter7 is the average daily fixed costs of RMGCs. Parameter8 is the average daily construction costs of matching facilities. Parameter9 is the average daily business costs of loading and unloading of RMGCs. Parameter10 is the average daily comprehensive costs of RMGCs. Line* is the line which has the loading or unloading business.

According to the results of table4, we can get the allocation and the costs shown as (14).

$$
\begin{aligned}
& 29898.9 \leq C_{g} \leq 38360.9 \\
& 3 \leq m \leq 4 \\
& 1 \leq r \leq 2 \\
& r, m \in Z
\end{aligned}
$$

As shown in table III and (14), we can see some allocation plans of RMGC. While $r=1$ and $m=3$, the flatcars entering the line could be loaded or unloaded directly without waiting. The total business time for finishing the loading and unloading is less than 24 hours. It means that the amount of RMGC allocated 
to this line could meet the demand of loading and unloading business.

While $r=2$ and $m=4$, the new added RMGC will be in idle state. That means if the amount of RMGCs is increasing, all new added equipments will be in idle state, too. Meanwhile, the power and electricity utilization rate is 0.8 under the allocation plan of $r=1$ and $m=3$. It is efficiency. And this maybe the optimal allocation plan.

(3) Calculation of objective function $\bar{T}$.

According to equation (5) and equation (12), with the constraints of equation (14), the objective function $\bar{T}$ could be calculated. The results of $\bar{T}$ are shown as table IV.

\begin{tabular}{ccccccc}
\multicolumn{1}{c}{ TABLE IV } \\
\multicolumn{10}{c}{ VALUES OF OBJECTIVE FUNCTION } & \multicolumn{1}{c}{ UNDER DIFFERENT ALLOCATION PLANS } \\
\hline \hline & 2 & 3 & 4 & 5 & 6 & 7 \\
line & RMGC & & $\min$ & $\min$ & $\min$ & $\min$ \\
\hline 1 & 3 & -- & 4.00 & -- & 0.00 & 4.00 \\
2 & 4 & $3: 1$ & 4.00 & 0.00 & 0.00 & 4.00 \\
\hline \hline
\end{tabular}

As illustrated in table IV, the parameter1 is the number of the loading and unloading lines of Chongqing railway container center station. Parameter2 is the total amount of RMGCs allocated to the station. Parameter3 is the ratio between the number of lines and the amount of the equipments allocated. Parameter4 is the average time of loading and unloading business for each flatcar in line1. And parameter5 has the same meaning as parameter 4 but in line2. Parameter6 is the average waiting time of flatcars on loading and unloading lines. Parameter7 is the average dwelling time of them.

According to the results of table $\mathrm{V}$, we can find that the average business time of loading and unloading of every flatcar is less than the interval time of flatcars arrival under the allocation plan of $r=1$ and $m=3$. That means the waiting time of flatcar for loading and unloading business on lines equals to zero. When adding the amount of RMGCs up to $m \geq 3$, the average rate of loading and unloading business for every flatcar is keeping at the level of 4 minutes. That is to say, adding more RMGCs could not reduce the average time of loading and unloading business for each flatcar. We obtained the optimal objective function $\bar{T}^{*}=4.00$ minutes.

Combined with $C_{g}$ in equation (14), we got the optimal allocation plan of RMGC in Chongqing railway container center station, shown as equation (15).

$$
\begin{aligned}
& \bar{T}^{*}=4.00 \text { minutes } \\
& C_{g}^{*}=29898.90 \text { Yuan } \\
& m^{*}=3 \\
& r^{*}=1
\end{aligned}
$$

Under the first term construction condition of the station, the optimal allocation of handling equipments is 3 with only one loading and unloading line. The optimal total daily comprehensive costs of handling equipments $C_{g}{ }^{*}$ are
29898.90Yuan. And the optimal average dwelling time on loading and unloading lines of railroad flatcars $\bar{T}^{*}$ is 4 minutes

\section{CONCLUSION}

The optimal allocation of handling equipments is in accordance with the equipment allocation facts of Chongqing railway container center station. The bi-objective optimal model is valid and can be used in equipment allocation in other railway container center stations.

\section{ACKNOWLEDGMENT}

Besides the sponsor and financial support acknowledgments above, the authors gratefully acknowledge Chinese Railway Container Transportation Co. Ltd for providing the data that form the basis for this paper.

\section{REFERENCES}

[1] Beijing Jiaotong University, Research on the key technology of multi-modal container express transit system, Beijing Jiaotong University, 2012.

[2] Yuan Ye, Research on Handling Equipment Allocation and Rail-Truck Connection Organization Based on Rail-Road Combined Transportation, Beijing Jiaotong University, 2012.

[3] Xin Song, Yinghong Li, and Ruoyu Wu, "Handling equipment selection and allocation in Kunming railway container center station", Railway transportation and economy, pp. 76-77, Aug. 2005.

[4] Ruihai Bai, Jun Yang, "Research on the handling equipment selection of railway container hub-station", Journal of railway engineering, pp. 77-82, Mar. 2002.

[5] Yuzhen Ma, "Selection of handling equipment of railway container center station", Railway transportation and economy, pp. 51-52, Oct. 2005.

[6] Jianyong Huo, Hui Xie, and Bo Hu, "Handling equipment selection in railway container hub and center station", Railway transportation and economy, pp. 68-69, June. 2006.

[7] Zongze Li, Wenming Cheng, Xiao Wu, and Zeqiang Zhang, "Research on equipment technology and layout in railway container center station", Journal of transportation engineering and information, pp. 101-105, Feb. 2005.

[8] Jian Liang, Wenming Cheng, and Min Zhang, "Simulation research for the loading and unloading business of train in railway container center station", Journal of system simulation, pp. 6290-6294, Oct. 2009.

[9] Linn R, Zhang C Q, "A heuristic for dynamic yard crane deployment in a container terminal", IEE transactions, pp. 161-174, Vol35, Feb. 2003.

[10] Peng Guo, "Modeling and simulation analysis of railway container logistics center", "Computer engineering and application", pp. 235-238, Vol.17, 2011.

[11] Zhong Wei, Research on schedule optimization of container yard logistics system, Beijing Jiaotong University, pp. 16-17, 2002

[12] Xingyan Yang, "Optimal investment scale of equipment in container yard based on the comprehensive cost model", Technology of ports, pp. 17-21, Feb. 1995.

[13] CF Daganzo, "The crane scheduling problem", Transportation Research, Part B, pp. 159-175, Vol.23, 1989.

[14] R.I.peterkofsky, CF Daganzo, "A branch and bound solution method for the crane scheduling problem", Transportation Research, Part B, pp. 159-172, Vol. 24, 1990.

[15] J.B.Tabernacle, "A study of the changes in performance of quayside container cranes", Maritime Policy and Management, pp. 115-124, Vol.22, 1995.

[16] J.Bose, Torsten Reines, Dirk Steenken, and Stefan Vob, "Vehicle dispatching at seaport container terminals using evolutionary algorithms", Proceedings of the Annual Hawaii International Conference on System Sciences, IEEE, Piscataway, 2000, pp.1-10.

[17] Lai,K.K, Lam,K, "A study of container yard equipment allocation strategy in Hong Kong", International Journal of Modeling and simulation, pp. 134-138, Vol. 14, 1994. 
[18] Kozan,E, Preston,P, "Genetie algorithm to schedule container transfers at multimodal terminals", International Transactions in Operational Research, pp. 311-329. June, 1999.

[19] CF Daganzo, "The crane scheduling problem", Transportation Research, Part B, pp. 159-175, Vol.23, 1989.

[20] Huijun Yang, "Research on handling equipment allocation optimization in container ports", Crane and transportation equipment, pp. 93-96, Dec. 2008.

[21] Pyung Hoi Koo, "Fleet sizing and routing for container transportation in a static environment", OR Spectrum, pp. 193-209, Vol.26, 2004.

[22] Mingjun Ji, "Allocation optimization of trailer in container yard based on simultaneous business", Journal of Dalian Marine University, pp. 47-49, Feb. 2010.

[23] Shuyan Zhang, Multi-objective optimization algorithm based on optimal constraints, Dalian Institute of Technology, 2010.

[24] Laumanns M, Thiele L, and Deb K, "Combining convergence and diversity in evolutionary multi- objective optimization", Evolutionary Computation, pp. 263-282, Oct. 2002.

[25] AG Hernández-Díaz, LV Santana-Quintero, and CA Coello Coello, "Pareto-adaptive $\varepsilon$-dominance", Evolutionary Computation, pp. 493-517, Vol.15, 2007.

Qi Zhang (Female), born in Lanzhou, Gansu Province, China, Sep. 1973. Dr. Zhang got her Bachelor degree of Transportation Engineering from Beijing Jiaotong University in 1996, Master degree in 1999 and Doctoral degree in 2012 of Transportation Engineering in Beijing Jiaotong University. The author's major fields of study are traffic and transportation planning and management, freight transportation, multi-modal transportation, and logistics.

Her work experience include: from 2006 to now, associate professor in School of Traffic \& Transportation, Beijing Jiaotong University; from 2001 to 2006, lecturer; from 1999 to 2001, teaching assistant. Her research interests are freight transportation, intermodal transportation, and logistics. 